\title{
A review on the sustainability of solar home system for rural electrification
}

\author{
Andrew Scott-George ${ }^{\mathrm{a}}$, Chong Li ${ }^{\mathrm{a} *}$, Yee Kwan Tang ${ }^{\mathrm{b}}$, Shimul Sahac, Muhammad Tareq Bin Alic, Aong Marma ${ }^{\mathrm{d}}$, Sudip \\ $\mathrm{Pal}^{\mathrm{e}}$ \\ a James Watt School of Engineering, University of Glasgow, United Kingdom \\ ${ }^{\mathrm{b}}$ Adam Smith Business School, University of Glasgow, United Kingdom \\ 'SEMWaves Ltd, United Kingdom \\ dJ\&C Impex Ltd, Bangladesh \\ ${ }^{\mathrm{e}}$ Chittagong University of Engineering and Technology, Bangladesh
}

\begin{abstract}
For the past 20 years, the solar home system (SHS) has been the dominant means of facilitating energy access for the poorest members of rural communities in the developing world. On paper, SHSs appear to be a sound solution to the difficulties in providing affordable and stable electricity to those likely to be left behind by other approaches. Indeed, evidence to date suggests that use of SHSs has accelerated the global electrification rate. However, there is mounting evidence that the long-term success rate of these systems and their effect on recipient communities is incredibly varied. Prompted by the results of a survey that uncovered widespread negative experiences amongst SHS users in rural Bangladesh, this study presents the results of a systematic literature review into the existing case studies of global SHS deployments. The search terms and websites used are presented clearly alongside the criteria used to filter results. Having identified a lack of long-term case studies the papers obtained are used to extract insights on the evolving strategy of SHS distribution, the mechanisms of SHS failure and crisis posed therein, and the most exciting areas of research for ensuring that when communities become electrified in future, they stay so. A three-element framework is offered for understanding SHS sustainability; community engagement, affordability, and appropriate maintenance infrastructure.
\end{abstract}

\section{Introduction}

\subsection{Background}

\subsubsection{Electrification in the $21^{\text {st }}$ Century}

Access to electricity is the $7^{\text {th }}$ of the United Nations Sustainable Development Goals (UN SDGs or SDGs). Electrification remains a difficult challenge in many parts of the world, particularly prevalent in rural areas of the global south. In these rural communities, low incomes and long distances from urban centres often make grid extension prohibitively expensive $(\$ 42,000 / \mathrm{km}$ in Bangladesh [1]). Islanded village-level microgrids are perhaps the best chance for this type of community to receive anything approaching grid quality electricity, but as in the case of grid extension the economic conditions are not always present to justify construction.

\subsubsection{The Solar Home System}

An 'energy ladder' model has been proposed to describe energy access development as a progression through multiple tiers. As a 'first step on the energy ladder' in low resource, remote communities, one technology prevails: the solar home system. SHS, as shown in Fig. 1, describes a small, modular energy unit essentially consisting of a solar panel, battery and charge controller. SHSs of the type used in developmental applications are most often designed to power a small array of DC appliances predominantly lightbulbs - for up to several hours a day.

As a minimally invasive, low cost, and easily transportable energy solution, SHSs are a highly attractive tool in bringing basic electricity to low income rural communities and as a result they have seen widespread deployment. Although it is hard to estimate exactly the number of SHS in use around the world, 2018 figures were around 4.4 million [2] and almost 1 million SHSs and other small solar-based products had been sold within the first half of 2020 alone [3].

\subsubsection{Limitations of SHSs and nature of field}

Despite the proven potential of these systems, SHS deployment efforts are not always successful. It is important that promoters of SHSs are fully informed about the potential consequences of using these systems,

* Corresponding author: chong.li@glasgow.ac.uk 
and literature has often been an essential tool in extracting insights of practical relevance from SHS experiences, both positive and negative [4].

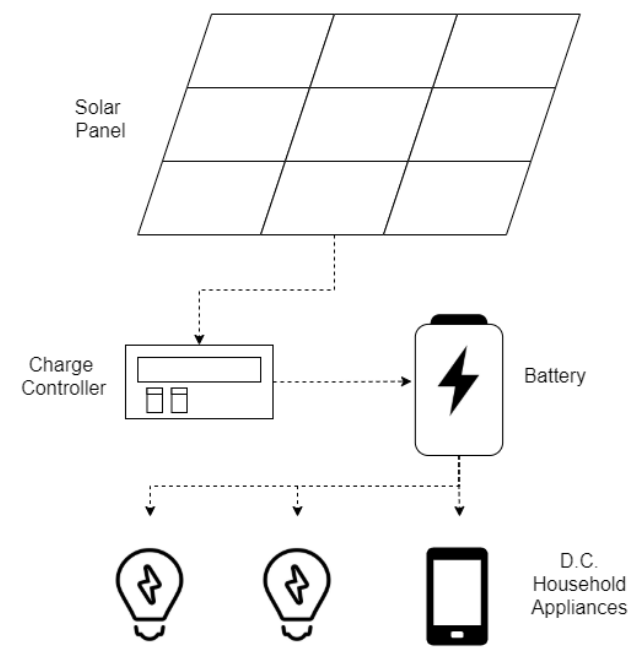

Fig. 1. SHS Diagram.

Methods of SHS research typically belong to one of three families; surveys concerning statistical analysis of primary data gathered from SHS users and stakeholders $[5,6]$, case studies blending quantitative and qualitative methods to comment on the experience of SHSs in a particular country or set of circumstances, often in a comparative context and over a longer period of time than a survey paper $[7,8]$ or literature reviews that synthesise existing publications to answer specific questions or make comments on broad observations $[9,10,11,12]$. Mixedmethods papers are also common $[13,14]$ and it is occasionally difficult to distinguish between 'surveys' and 'case studies' which both handle primary data.

This study positions itself within the last of the three main methods, using a literature review to explore the role of SHSs in current electrification efforts and the best practices therein. The literature review has been systematised as best as possible, however due to the limited resources and timeframe at hand it was not possible to exhaust the extant literature. Thus, the study is best categorised as a 'literature review with systematic techniques' or a 'semi systematic review' [15].

The study itself was prompted by a preliminary survey into SHS user experiences in rural Bangladesh [16]. This survey will be discussed to provide a motivational background to the paper before proceeding with the methodology, results, and a discussion of the core findings. It is hoped that readers will be left with an understanding of the knowledge limitations in the research on SHS usage, an overview of the best practices in the field, and several avenues for further research.

\subsection{Bangladesh Survey}

Bangladesh has devoted an immense amount of its national resources towards achieving rural electrification through off-grid solar technologies, particularly SHSs [17]. Infrastructure Development Company Limited (IDCOL) reports that 4.13 million SHSs had been deployed in Bangladesh as of January 2019 [18], a significant proportion of the global level estimated in the previous year.

To explore experiences with SHSs in rural Bangladesh, in 2019 we (the authors) conducted a survey amongst 7 villages in the Chittagong Hill Tracts region [18]. SHSowning households were interviewed to gain an understanding of how they had acquired SHSs, how much they had paid and for what type of system, and their opinion of the energy service they were now receiving.

Of 46 respondents, almost all had strong complaints regarding their SHSs - most found their systems to be unreliable, expensive, and incapable of providing sufficient energy. Many were still paying off their units several years after initial purchase, with battery replacements only a few more years away. Dissatisfaction was prevalent even amongst the minority of respondents who had received their units by donation, and almost all maintained a desire for better quality energy.

When considering these findings against the Bangladesh governments planned \$26 million investment in SHSs [19] it can appear as though there is a fundamental mismatch between the realities of SHS usage and the perceptions of well-intentioned promoters. A reflection on the role of SHSs in modern electrification strategy is needed, as well as knowledge of best practices.

\section{Methodology}

\subsection{Search Method}

Three databases were used to conduct the search: Web of Science, Google Scholar, and Scopus. The search terms used are given in the Table 1.

Table 1. Search terms

\begin{tabular}{|l|l|}
\hline \multirow{3}{*}{$\begin{array}{l}\text { ("Solar Home System" } \\
\text { OR "Solar Home }\end{array}$} & "Literature Review" \\
\cline { 2 - 2 } Systems) AND... & "Experience" OR \\
\cline { 2 - 2 } & "Experiences" \\
\cline { 2 - 2 } & "Survey" OR "Surveys" Study" OR "Case \\
& Studies" \\
\cline { 2 - 2 } & "Year" OR "Years" \\
\cline { 2 - 2 } & "Lessons" OR "Insights" \\
\cline { 2 - 2 } & "Development" \\
\cline { 2 - 2 } & "Market" \\
\cline { 2 - 2 } & "Failure" OR "Success" \\
\cline { 2 - 2 } & 'Energy Ladder' \\
\hline
\end{tabular}

\subsection{Sorting Results}

Papers were initially selected based on the following criteria:

1) Does the study refer to experiential data regarding SHS usage?

2) Does the study analyse the success of one or more SHS deployments?

3) Does the study consider the developmental impacts of SHSs?

4) Does the study focus on the global south? 
Once an initial set of papers had been obtained, preference was given to those from high impact journals and to those published more recently. This allowed an initial set of 94 relevant papers to be reduced to a manageable 40 for deeper analysis. It was acknowledged that papers using alternative nomenclature to "Solar Home System(s)" may have been neglected but given the prevalence of the term and the extensive results found it was not considered necessary to expand the search further.

\section{Results}

\subsection{State of Literature}

The overall literature content regarding SHS experience was found to be extensive with a few notable gaps. As may be expected from the increasing penetration of SHS units worldwide, the number of papers focusing on the subject matter has expanded with time. More attention was directed at studies within the past ten years (20112021) than earlier ones (prior to 2011) that may reference redundant technological landscapes and discontinued practices. The selection of 2011 is arbitrary; SHS deployment figures are so difficult to estimate that any meaningful milestone could not be credibly used. Of the final 40 papers, 21 were published in or after 2011.

\subsection{Lack of Long-term Knowledge}

It became apparent during the literature review that there appears to be a gap in longer-term studies of SHS deployments. Longer-term was taken here to mean $>6$ years after initial deployment, after which point batteries should have been replaced [20] thus testing the resilience of projects to cyclic financial strains - an important aspect of long-term sustainability. Of the 40 papers analyzed, as shown in Fig. 2, only $15 \%$, that is 6 , focused on long-term SHS usage. Half of the surveyed papers were reported 6 years and under. The remaining 35\% papers did not specify period since units being installed.

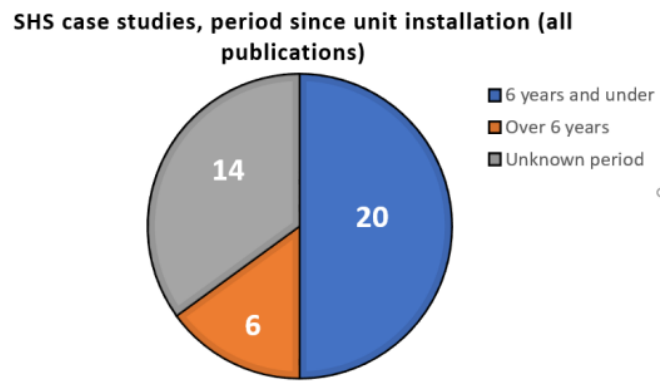

Fig. 2. SHS case studies, period since unit installation (all publications).

It might be expected that recent papers would be better in this regard, benefitting from a wider range of longestablished SHSs, however in fact Fig. 3 shows that long term studies were particularly lacking in publications since 2011. Only 2 out of 21 papers are for over 6 years. This indicates that the sustainability of contemporary SHS deployment practices is yet to be fully explored. The longest-term monitoring studies were perplexingly some of the oldest discovered [21, 22].

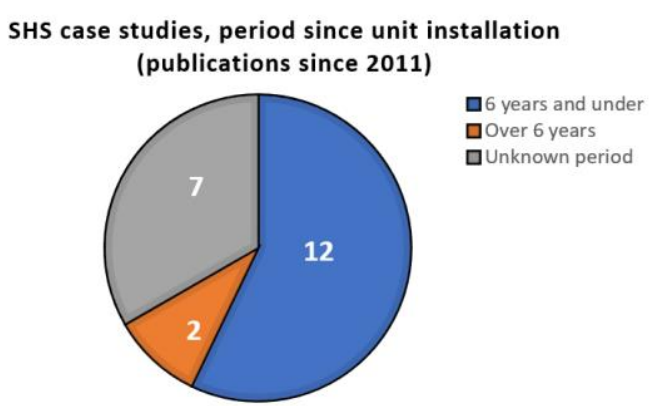

Fig. 3. SHS case studies, period since unit installation (publications since 2011).

\subsection{Summary}

Despite an informative volume of papers discussing the successes and failings of SHS globally, the literature suffers from a distinct lack of long-term SHS user monitoring. This may in part be due to the difficulty of obtaining data from commercial distributors [23].

To express the insights derived from the literature review, a techno-economic perspective will be taken in the following sections. A reflection on the developmental contribution of SHSs is first given, after which the various factors that influence a successful deployment of SHSs are presented.

\section{Discussion}

\subsection{SHSs as a Tool of Sustainable Development}

SHSs have been seen to elicit improvements in quality of life [16], particularly education and reducing reliance on kerosene, an expensive and environmentally harmful fossil fuel [4]. However, this is not universal, and not necessarily a guarantee of user satisfaction. Similar negative experiences to those in our own survey have been documented elsewhere [24, 25, 26], and reviews of the contribution of SHSs to development have noted the mixed and contradictory opinions that prevail in the literature [27].

A common thread in the analysis of where SHSs have failed to match expectations is in the level of energy access actually provided by them. What is meant by electrification and energy access? By design, small SHSs can only provide DC electricity for specialised components, already limiting their developmental potential to in many cases the provision of lighting alone $[28,29]$. However, other authors argue that appliances are not the most important metric for assessing energy development [30]. Particular appliances aside, a Venezuelan study [31] found $2 \mathrm{kWh} /$ day to be the minimum level of energy access for end-user satisfaction - far above what a typical small SHS unit $(<100 \mathrm{Wp})$ can provide.

If this is so, and SHSs can only meet truly the most basic of needs, then their role as a long-term feature of development is questionable. It is well established that once a minimum level of energy access is present, this can rapidly expand $[29,5]$ and it is worth asking what happens 
to SHSs when they are no longer sufficient. If SHSs are to be a 'first step on the energy ladder', then there must be follow up to allow users to continue to increase their energy access level. This is often not the case [30] and the aforementioned lack of long-term monitoring means the reality is largely unknown.

Some authors point to the declining costs of microgrids as removing the need for SHSs as an intermediate step altogether [29], although the additional capital and risk embodied mean SHSs will still have a role for many years to come. There is evidence that SHSs are anyway used in parallel to microgrids, to reach the poorest members of a community and provide backup [32].

In summary, despite the presence of negative experiences with SHS it appears they still have a role to play in facilitating basic energy access until other options become available. For this reason, they are likely to remain an important part of rural electrification strategy in the global south for years to come. To that end, it is important to understand the best practices for their deployment. A discussion of findings in this vein is now presented.

\subsection{Three Factor Model: Cost, Community, and Continued Support}

In the analysis of the literature surveyed, success (or conversely risk) factors surrounding SHS deployments were able to be categorised under a three-element framework which is illustrated in Fig. 4. Of the surveyed papers, $100 \%$ made reference to low costs as a deciding factor in the viability of an SHS endeavour, with a further $73 \%$ referring to continued support through operations \& maintenance (O\&M) and $45 \%$ to community engagement and buy in.

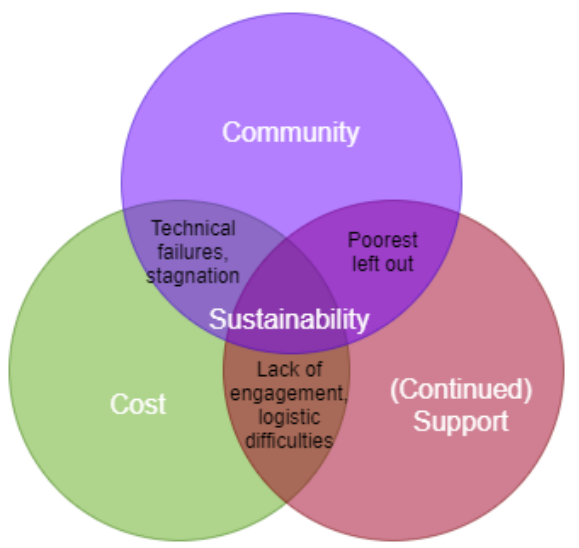

Fig. 4. SHS Sustainability Factors Diagram.

There are many lenses with which to view the factors influencing the success of a given SHS deployment effort. The three headings shown were found to be useful in broadly categorising where a project may have gone wrong as a catalyst for deeper discussion. The three elements of the model are very closely linked, and the 'risk factors' or 'success criteria' discovered in the literature were often best conceptualised through two elements in tandem.

\subsection{Creating Low Costs and Integrating Maintenance}

Many of the cost barriers prevalent at the inception of SHSs in the late 1980s - expensive, large, and short-lived batteries, malfunctioning charge controllers, and expensive, inefficient solar PV [33, 22, 21] - have been overcome with time. Cost reductions have reached the point that small SHSs cost only tens of dollars to purchase, at most a few months of the lowest of rural incomes.

Batteries have the most changes still to come; the traditional approach has been lead-acid (LA) due to low cost and a long history of use, but as costs continue to fall the longer lived, and more energy dense lithium-ion (LiIon) is increasingly prevalent in SHSs. The discussion of LA vs Li-Ion in off-grid solar and SHSs has been extensively covered $[20,34]$, and it has been seen that in most cases, Li-Ion is cheaper in the long run than LA. However, given that low upfront costs are of the utmost priority for low-income consumers, both LA and Li-Ion are likely to remain a feature of SHSs for the foreseeable future. The greater level of maintenance required and the shorted lives of LA batteries are likely to mandate a greater level of continued support in budget SHSs.

Affordability is not just a product of hardware costs; distribution business models are of equal importance. The provision of microfinance and loans at both the user and business level has allowed capital costs to be stretched over a manageable timeframe. Novel business models such as pay-as-you-go (PAYG), in which an energy service company (ESCO) rents solar systems out to end users, have transformed the relationship with SHSs as full ownership is no longer a necessity $[35,36]$.

\subsection{Forms of Continual Support}

At least three elements of continued support were identified for SHS programs to aid sustainable development: subsidy and policy support from governments and towards ESCOs, O\&M of the SHS units themselves, and opportunities for progression from SHSs to more permanent means of electrification.

The short lifetimes of both batteries (2-5 years for LA) and appliances present in SHSs can often come as a surprise to users who have been marketed a 'permanent' solution to their energy needs. Communication of maintenance requirements and proper usage is integral to managing user expectations, and there are often calls for providing technical training to users [35, 37, 38, 25]

For maintenance to take place, there is also the need for supply chains that can provide parts and technical labour affordably and promptly. Setting up this kind of infrastructure in remote regions is no easy task. The same business models that remove the financial burden of owning a SHS (PAYG) also place maintenance responsibility in the hands of ESCOs that have more resources available to them $[36,8,35]$ and may be better equipped to provision parts and employ skilled labour.

Examples from South America [13] demonstrate that supporting local entrepreneurs who benefit from community connections and local knowledge is one of the best ways of embedding support SHS in a society. While 
earlier papers have called for better technical quality regulation in SHSs markets, more recent authors have taken the stance that these impositions can act as a barrier to local entrepreneurs who may better develop robust supply chains through the sale of mixed-quality and improvised goods [39].

\subsection{Who should be responsible for SHSs?}

Governments and non-governmental organisations (NGOs) have traditionally been the main drivers of electrification via SHS, as their human-centric interests most directly align with a field which is largely unprofitable. And yet, examination of successful enterprises such as Grameen Shakti in Bangladesh has yielded the insight that the intrinsic need to 'swim or sink' in the private sector may yield practices with long-term sustainability in mind [40]; put plainly, private sector involvement in SHS distribution is a strong predictor of success [41]. This is most harmoniously achieved through 'public-private-partnerships' [42, 43, 13], which allow governments to align the efficiency and local knowledge of businesses with developmental interests through subsidies, local monopolies, and institutional support.

\section{Conclusion}

The utility of SHSs in providing rapid access to basic levels of electricity is reinforced by the literature. However, experiences are widely varied. Where SHSs are used as a more permanent development tool, a variety of considerations must be made to ensure the benefits they bring are part of a larger narrative of sustainable development and progressive electrification.

A gap in the long-term knowledge of SHS experience was identified. Although it can be laborious for surveyers to document the duration of time that their respondents have owned SHS for, this is an integral metric for understanding the developmental impact of SHSs on two counts. Firstly, if the energy ladder model is to be believed then data is necessary to examine whether SHSs have indeed acted as a 'step on the ladder' towards more permanent forms of access or whether users are stuck at the SHS level for extended periods of time. Establishing a timeframe for user experiences of SHS could help to better plan their role in future electrification efforts. Given that certain aspects of SHSs such as battery replacement do not present until several years after deployment, it would benefit the discussion if there was more evidence for the resilience of SHS projects to these successive waves of costs.

Within the examined literature, a three-element framework was developed to conceptualise the sustainability of a given SHS deployment. Low costs are required for users to afford systems, sufficient levels of community engagement are required for units to be well adopted and integrated, and systems for the continual support of SHS units must be cultivated to provide O\&M to existing systems as well as progress to preferable means of energy access.

\section{References}

1. S. K. Nandi et al., Energy, 35(2010)

2. GOGLA, "Lighting Global: Off-grid Solar Market Trends Report 2018” (2018)

3. GOGLA, "Global Off-Grid Solar Market Report Semi-Annual Sales and Impact Data"(2020)

4. M.A. Hossain Mondal, Renewable Energy, 35(2010)

5. M. Gustavsson, Renewable Energy, 32(2007)

6. X. Yuan et al., Energy Policy, 39(2011)

7. B.K. Sovacool and A.L. D'Agostino, Prog. Dev. Stu.,12(2012)

8. M.S. Barry and A. Creti, Energy Economics, 90(2020)

9. V. Kizilcec and P. Parikh, Ener. Sus. Dev., 58(2020)

10. A.A. Eras-Almeida and M Egido-Aguilera, Sustainability, 12(2020)

11. F.D.J. Nieuwenhout et al., Prog. Phot., 9(2001)

12. C. Brunet et al., Ren. Sus. Ene. Rev., 96(2018)

13. A.A. Eras-Almeida et al., Sustainability, 11(2019)

14. L. Joshi et al., World Development, 122(2019)

15. H. Snyder, J. Business Research, 104(2019)

16. C. Li et al., University of Glasgow internal report (2019)

17. T. Urmee and D. Harries, Renewable Energy, 36(2011)

18. IDCOL, "Solar", 2903 2021. [Online]. Available: http://idcol.org/home/solar.

19. PV Magazine, "Bangladesh to install another 40,000 free solar home systems," PV Magazine, 16 July 2020. [Online]. Available: https://www.pvmagazine.com/2020/07/16/bangladesh-to-installanother-40000-free-solar-home-systems/. [Accessed 30 March 2021].

20. S. Anuphappadorn et al., Energy Procedia, 56 (2014)

21. M. Djamin et al., World Renewable Energy Conference VI(2000)

22. A. Reinders, Ren. Sus. Ene. Rev., 3 (1999)

23. M.C. Mapako et al., Domestic Use of Energy Conference (2004)

24. K. Mala, Renewable Energy, 34 (2009)

25. C. L. Azimoh et al., Applied Energy, 136 (2014)

26. S.M. Rahman and M.M. Ahmad, Energy Policy, 63 (2013)

27. X. Lemaire, WIREs Ene. Env., 7 (2018)

28. C.L. Azimoh et al., Applied Energy, 155 (2015)

29. D. Chattopadhyay et al., The Electrictiy Journal, 28 (2015)

30. I. Bisaga and P. Parikh, Ene. Res. Soc. Sci., 44 (2018)

31. López-González et al., Energy Policy, 129 (2019)

32. E. Harrington et al., Ren. Sus. Ene. Rev., 119 (2020)

33. S. Dhundhara et al., Ene. Conv. Mana., 177 (2018)

34. P. Yadav et al., Ene. Sus. Dev., 22 (2020)

35. G. Adwek et al., Env, Dev, Sus, 22 (2020) 
36. M. Barman et al., Ene. Sus. Dev., 38 (2017)

37. H. Holtorf et al., Ren. Sus. Ene. Rev., 50 (2015)

38. A.C. Groenewoudt et al., Ene. Sus. Dev., 58 (2020)

39. B. Sovacool et al., Energy, 36 (2010)

40. D. Palit., Ene. Sus. Dev., 17 (2013)

41. A. Newcombe et al., Ene. Sus. Dev., 38 (2017)

42. B. Sovacool., Ene. Strat. Rev., 1 (20130) 